\title{
Strongly aligned gas-phase molecules at Free-Electron Lasers
}

\author{
Thomas Kierspel ${ }^{1,2}$ Joss Wiese, ${ }^{1}$ Terry Mullins, ${ }^{1}$ \\ Joseph Robinson, ${ }^{3}$ Andy Aquila, ${ }^{3}$ Anton Barty, ${ }^{1}$ \\ Richard Bean, ${ }^{1,4}$ Rebecca Boll, ${ }^{5}$ Sébastien Boutet, ${ }^{3}$ \\ Philip Bucksbaum, ${ }^{3,6,7}$ Henry N. Chapman, ${ }^{1,2,8}$ \\ Lauge Christensen, ${ }^{9}$ Alan Fry, ${ }^{3,6}$ Mark Hunter, ${ }^{3}$ \\ Jason E. Koglin, ${ }^{3}$ Mengning Liang, ${ }^{3}$ Valerio Mariani, \\ Andrew Morgan, ${ }^{1}$ Adi Natan, ${ }^{6}$ Vladimir Petrovic, ${ }^{6}$ \\ Daniel Rolles ${ }^{5,10}$ Artem Rudenko, ${ }^{10}$ Kirsten Schnorr, ${ }^{11}$ \\ Henrik Stapelfeldt, ${ }^{9}$ Stephan Stern, ${ }^{1}$ Jan Thøgersen, ${ }^{9}$ \\ Chun Hong Yoon, ${ }^{1,4}$ Fenglin Wang, ${ }^{1,6}$ Sebastian Trippel, ${ }^{1}$ \\ and Jochen Küpper ${ }^{1,2,8}$ \\ E-mail: jochen.kuepper@cfel.de \\ website: http://www.controlled-molecule-imaging.org \\ 1 Center for Free-Electron Laser Science, DESY, 22607 Hamburg, Germany \\ 2 Center for Ultrafast Imaging, University of Hamburg, 22761 Hamburg, \\ Germany \\ 3 LCLS, SLAC National Accelerator Laboratory, Menlo Park, CA, 94025, USA \\ 4 European X-ray Free Electron Laser (XFEL) GmbH, 22761 Hamburg, \\ Germany \\ 5 Deutsches Elektronen-Synchrotron (DESY), 22607 Hamburg, Germany \\ 6 SLAC National Accelerator Laboratory, PULSE Institute, Stanford, CA, \\ 94305, USA \\ 7 Department of Physics, Stanford University, Stanford, CA 94305, USA \\ 8 Department of Physics, University of Hamburg, 22761 Hamburg, Germany \\ 9 Department of Chemistry, Aarhus University, 8000 Aarhus C, Denmark \\ 10 Department of Physics, Kansas State University, Manhatten, KS, 66506, \\ USA \\ 11 Max Planck Institute for Nuclear Physics, 69117 Heidelberg, Germany
}

\begin{abstract}
We demonstrate a novel experimental implementation to strongly align molecules at full repetition rates of free-electron lasers. We utilized the available in-house laser system at the coherent x-ray imaging beamline at the Linac Coherent Light Source. Chirped laser pulses, i. e., the direct output from the regenerative amplifier of the Ti:Sa chirped pulse amplification laser system, were used to strongly align 2,5-diiodothiophene molecules in a molecular beam. The alignment laser pulses had pulse energies of a few mJ and a pulse duration of $94 \mathrm{ps}$. A degree of alignment of $\left\langle\cos ^{2} \theta_{2 \mathrm{D}}\right\rangle=0.85$ was measured, limited by the intrinsic temperature of the molecular beam rather than by the available laser system. With the general availability of synchronized chirped-pulse-amplified near-infrared laser systems at short-wavelength laser facilities, our approach allows for the universal preparation of molecules tightly fixed in space for experiments with x-ray pulses.
\end{abstract}

28 July 2015

Submitted to: J. Phys. B: At. Mol. Phys.

Keywords: FEL, Gas Phase, Alignment 


\section{Introduction}

Imaging the structural dynamics of gas-phase molecules using approaches such as photoelectron imaging in the molecular frame [1 3], high-harmonic-generation spectroscopy [4, 5], laser-induced electron diffraction [6. 7], electron diffraction [8, 9], or x-ray diffraction [10] [12] is much improved or is only possible by fixing the molecules in space [8, 10,14. Various approaches to align or orient molecules have been demonstrated, ranging from state-selection 15 over brute-force orientation [16] to laser alignment [13]. Generally, laser alignment has been implemented in two different regimes, namely, using short laser pulses to "impulsively" create coherent rotational wavepackets that generate alignment some time after the laser pulse [17, 18] or using long laser pulses to "adiabatically" create pendular states that are strongly aligned during the laser pulse [19]. In the former approach, pulses from standard commercial Ti:Sapphire (TSL) laser systems can be utilized at repetition rates up to several kilohertz, whereas in the latter approach, traditionally, injection seeded Nd:YAG lasers were employed, with hundreds of millijoules of pulse energy, limited to a few $10 \mathrm{~Hz}$. Through the addition of weak dc electric fields, strong orientation can be achieved in both scenarios 20 22. Three-Dimensional (3D) alignment or orientation was also demonstrated [20, 22, 26]. It was shown that the degree of alignment necessary for molecularframe diffractive imaging is very high [11, 14, 27] and, for complex molecules, could not be achieved with impulsive alignment approaches. Generally, the degree of alignment can be improved through higher laser-field intensities, limited by the onset of ionization, or colder samples 28], possibly achieved through state selection 20- 22.

The intermediate-pulse-duration regime, for which the alignment laser pulse duration is shorter than the lowest rotational period of the molecule, but cannot be considered impulsive anymore, had previously been analyzed theoretically [29 31]. While the laser pulse is still on, the molecules act similar to the adiabatic alignment case, whereas after the pulse periodic revivals can be observed 29]. Recently, this mesobatic regime was explored experimentally and it was realized that degrees of alignment comparable to the adiabatic limit can be achieved with much shorter and weaker laser pulses, e. g., from commercial chirped-pulse-amplified (CPA) TSL systems [32, 33. While the nonadiabatic interaction creates interesting dynamic phenomena such as the observation of pendular motion [33], this approach does provide strongly aligned samples of complex molecules [32]. Moreover, it requires only moderately long pulses on the order of 100 ps with moderate pulse energies, i.e., a few mJ, which are available as output from the standard laser systems

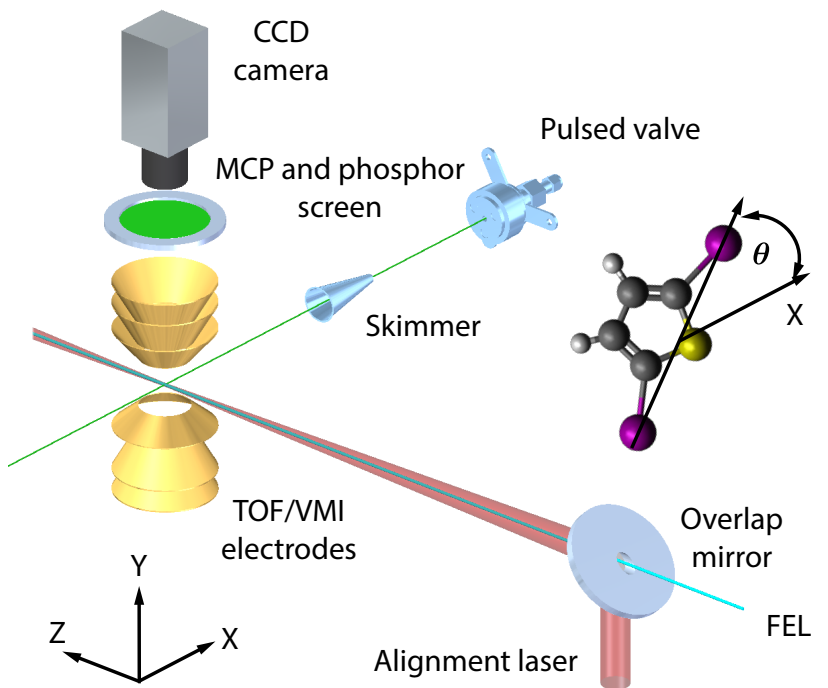

Figure 1. Sketch of the experimental setup showing the pulsed molecular beam valve, the laser beam path of the alignment laser (red) and the free-electron laser (cyan), and a holey mirror to collinearly overlap both beams. The detection system consists of an ion-imaging setup for time-of-flight measurements and velocitymap imaging onto a micro-channel plate, phosphor screen, and a CCD camera. In the inset the definition of $\theta$ is depicted, it is the angle between the laser polarization, i. e., the $X$ axis, and the most polarizable axis of the molecule, i. e., the iodine-iodine axis.

available at $\mathrm{x}$-ray laser facilities.

Here, we demonstrate the successful implementation of intermediate-pulse-duration regime, strong alignment of the prototypical asymmetric rotor molecule 2,5diiodothiophene $\left(\mathrm{C}_{4} \mathrm{H}_{2} \mathrm{I}_{2} \mathrm{~S}\right)$ in a molecular beam at the Coherent X-ray Imaging (CXI) [34, 35] endstation of the Linac Coherent Light Source (LCLS) at the SLAC National Accelerator Laboratory (SLAC). Previous experiments with fixed-in-space molecules at free-electron lasers (FELs) have exploited both, impulsive and adiabatic alignment, for instance, in ion imaging [36, 37], electron imaging 3, 38, 40, or x-ray diffraction experiments [10, 41]. Our approach allows one to combine the strong alignment achieved in adiabatic alignment approaches [3, 10, 39, 41] with the utilization of x-ray pulses at the full repetition rate of the LCLS [37, 38]. This allowed to perform experiments at $120 \mathrm{~Hz}$, comparable to previously demonstrated intermediate regime alignment at $1 \mathrm{kHz}$ in table-top experiments [32]. For comparison, we also provide measurements for the degree of alignment from experiments conducted in our laboratory at the Center for Free-Electron Laser Science (CFEL) [32] during the beam time preparation.

\section{Experimental Setup}

Figure 1 shows a sketch of the experimental setup at LCLS. A trace of 2,5-diiodothiophene was coexpanded in 80 bar helium into the vacuum by a pulsed valve [42, 
operated at $75^{\circ} \mathrm{C}$, and at a repetition rate of $120 \mathrm{~Hz}$ synchronized to the LCLS. The molecular beam was skimmed $8 \mathrm{~cm}$ downstream the nozzle using a $3-\mathrm{mm}-$ diameter skimmer. This resulted in a $5.2 \mathrm{~mm}$ molecular beam (full width at half maximum, FWHM) in the interaction zone.

Near-infrared (IR) laser pulses were produced in a CPA TSL system (Coherent) with a central wavelength of $800 \mathrm{~nm}$. The laser system is operated at and synchronized to the repetition rate of LCLS. The chirped pulses out of the regenerative amplifier were split using a beam splitter with a reflectivity of $90 \%$. The reflected pulses, which were used to align the molecules [32, 33, had a pulse energy of $3.3 \mathrm{~mJ}$ and a pulse duration of $94 \mathrm{ps}$ (FWHM). The transmitted pulses were compressed using a standard grating-based compressor to a pulse duration of $55 \mathrm{fs}$ (FWHM) at a pulse energy of $250 \mu \mathrm{J}$. The compressed pulses multiply ionize the molecules through strongfield ionization, resulting in Coulomb explosion; in all experiments these compressed pulses were linearly polarized, with the polarization perpendicular to the long axis of polarization of the alignment laser pulses (vide infra). Both arms were spatially and temporally recombined before entering the vacuum system. This, in combination with ion imaging (vide infra), allows to characterize and optimize the degree of alignment without the FEL beam.

The short pulses were also utilized to measure the pulse duration of the alignment laser pulses through spectral interference. The frequency components of the chirped pulse arrive sequentially, whereas all frequencies in the compressed pulse are temporally overlapped. At finite spectrometer resolution, interference in the combined spectrum is only visible at those wavelength components of the alignment pulse that are temporally overlapped, or nearly overlapped, with the compressed pulse. The strongest interference is visible at the wavelength that temporally coincides with the compressed pulse. A delay line was used to change the relative timing between the chirped alignment and the compressed pulses. Thus, monitoring the spectrum in dependence of the delay between these two pulses provided information on the pulse duration of the chirped pulse.

To reach sufficiently high intensities to align the molecules, a telescope was used to expand both beams by a factor of three before being focused into the chamber with a 1-m-focal-length lens. A holey mirror was used to spatially overlap the TSL pulses with the FEL beam path, losing $20 \%$ of the near-infrared laser power. The hole had a diameter of $2 \mathrm{~mm}$ and the FEL beam was guided through the hole to achieve a collinear propagation of both beams. The alignment laser focus size was $45 \mathrm{\mu m}$ (FWHM) leading to an estimated peak intensity at the focus on the order of $1 \cdot 10^{12} \mathrm{~W} / \mathrm{cm}^{2}$. The FEL beam size was estimated to $12 \mu \mathrm{m}$ in the horizontal and $3 \mu \mathrm{m}$ in the vertical axis. The different beam diameters made the setup more stable against beam pointing imperfections and ensured that only well aligned molecules were probed with the FEL. The alignment laser pulse and the FEL/TSL pulses were spatially and temporally overlapped in order to maximize the degree of alignment; the exact timing was not critical 32 .

The alignment laser was elliptically polarized with an aspect ratio of 3:1 in order to obtain 3D alignment. The major axis was parallel to the micro-channel plate (MCP) and phosphor screen surface. That way the most polarizable axis of 2,5-diiodothiophene, i. e., the iodineiodine axis, was confined to the $X$ axis as can be seen in Figure 1. The degree of alignment was probed using $\mathrm{x}$-ray pulses with a pulse duration of approximately 70 fs (FWHM), a photon energy of $9.5 \mathrm{keV}$, and a pulse energy of approximately $0.64 \mathrm{~mJ}\left(4.2 \cdot 10^{11}\right.$ photons, beam line transmission $80 \%$, focussing mirror transmission $40 \%$ ) in the interaction zone. X-ray absorption is largely localized at one of the iodine atoms, followed by Auger cascades and intramolecular charge redistribution leading to Coulomb explosion of the molecule. The ions were accelerated by the electric field of the spectrometer (Figure 1) towards a position sensitive detector consisting of a MCP and a phosphor screen (Photonis). A CCD camera (Adimec Opal), operating at the FEL repetition rate, recorded single-shot ion distributions visible as light spots on the phosphor screen. A real time online monitoring software, implementing a peak finding [43] and centroiding 44, 45 algorithm, was used to identify and localize single ion hits on the detector. By measuring the transverse velocity distribution of the iodine cations, the degree of alignment is evaluated by determining $\left\langle\cos ^{2} \theta_{2 \mathrm{D}}\right\rangle \neq$ where $\theta$ is defined as indicated in Figure 1 and $\theta_{2 \mathrm{D}}$ is defined as indicated in Figure 3

The detection system allowed to switch between a time-of-flight (TOF) [46] mode to determine the various fragment masses and a velocity map imaging (VMI) 47] mode to determine the ion angular distribution. In the VMI mode, a fast high-voltage switch (Behlke) was used to gate the phosphor screen on a single iodine charge state determined from the TOF mass spectrum (MS).

The experimental details for the comparison measurement performed at the CFEL are described in reference 32. In short, the molecular beam was triply skimmed before it entered a VMI spectrometer where it was crossed by the alignment and probe laser pulses. The distance from the valve to the interaction zone

$\ddagger$ The two-dimensional degree of alignment is defined as $\left\langle\cos ^{2} \theta_{2 \mathrm{D}}\right\rangle=\int_{0}^{\pi} \int_{0}^{r_{\max }} \cos ^{2}\left(\theta_{2 \mathrm{D}}\right) f\left(\theta_{2 \mathrm{D}}, r_{2 D}\right) d r_{2 D} d \theta_{2 \mathrm{D}}$, where $f\left(\theta_{2 \mathrm{D}}, r_{2 D}\right)$ is the normalized $2 \mathrm{D}$ projection of the probability density. 


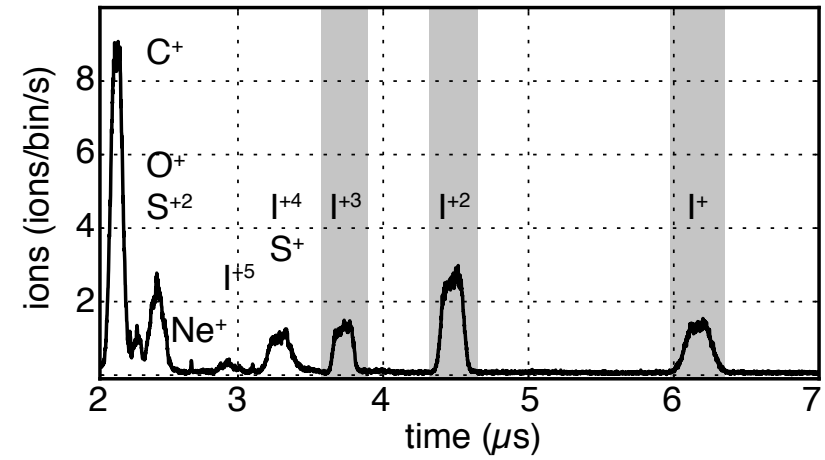

Figure 2. Time-of-flight mass spectrum of the ionic fragments produced in the Coulomb explosion of 2,5-diiodothiophene following absorption of a $9.5 \mathrm{keV}$ x-ray photon. The gray areas indicate the gating windows for the VMI spectrometer used to determine the angular distribution for each fragment in Figure 3

was approximately a factor of 3.5 longer than at the LCLS. The sample reservoir was heated to $40^{\circ} \mathrm{C}$ and a stagnation pressure of 90 bar helium was applied. The alignment and probe laser pulses were provided by splitting the output of an amplified femtosecond TSL system into two parts. The alignment pulses were stretched, or, more accurately, overcompressed. This resulted in negatively chirped pulses with a duration of $485 \mathrm{ps}$ (FWHM) and focused to a beam waist of $36 \mu \mathrm{m}$ times $39 \mu \mathrm{m}$ (FWHM). At a maximum pulse energy of $7.4 \mathrm{~mJ}$ a focus peak intensity of $9.6 \cdot 10^{11} \mathrm{~W} / \mathrm{cm}^{2}$ can be estimated. The temporal profile of the alignment pulse had a trapezoid-like shape, i. e., fast rising time (100 ps), longer plateau (400 ps), and fast falling time (100 ps). The alignment laser was elliptically polarized with an aspect ratio of $3: 1$. The probe pulses were compressed to $30 \mathrm{fs}$ and focused to $27 \times 28 \mu^{2}$ (FWHM).

\section{Experimental Results}

\subsection{Results from experiments at $L C L S$}

Following the absorption of a $9.5 \mathrm{keV}$ x-ray photon the 2,5-diiodothiophene molecules charge up and fragment through Coulomb explosion. Figure 2 shows a timeof-flight (TOF) mass spectrum (MS) build up as a histogram of events from several thousand single-shot mass spectra. Clearly separated peaks for singly to triply charged iodine ions are visible, while the peaks for higher charge states of iodine ions overlap with other fragments. Based on this TOF spectrum, we have recorded angular distributions of the $\mathrm{I}^{+}, \mathrm{I}^{+2}$, and $\mathrm{I}^{+3}$ fragments with the VMI spectrometer by gating the detector for the appropriate arrival times, as indicated by the gray areas in Figure 2 .

In Figure 3 a sum of several thousand individuallyprocessed VMI images for several iodine cation fragments of 2,5-diiodothiophene upon ionization with

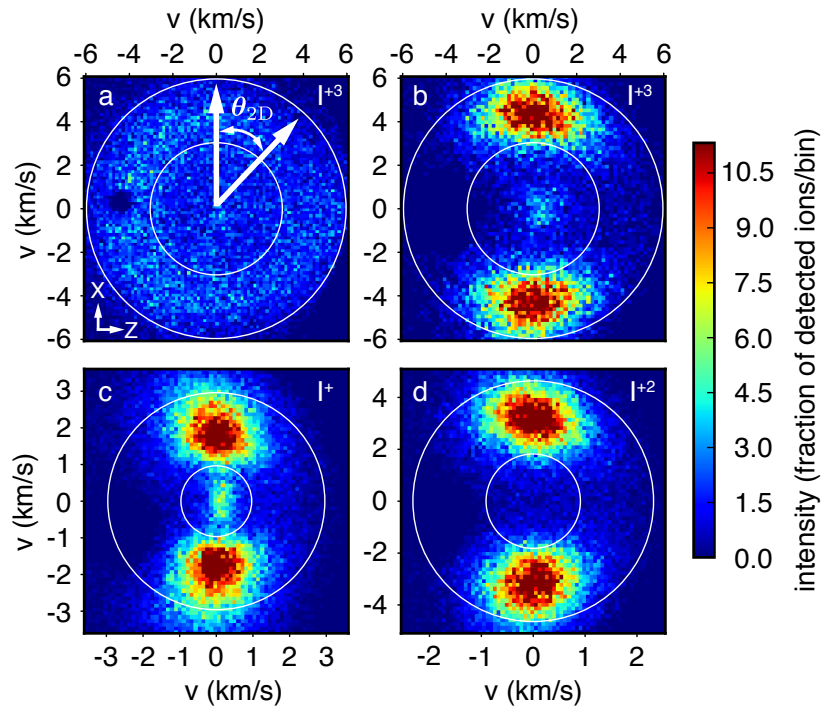

Figure 3. VMI images for (a) isotropic and (b-d) aligned iodine cations. The area between the inner and outer circle was used to determine the degree of alignment. The laboratory framework and the definition of the angle $\theta_{2 \mathrm{D}}$ is shown in a). The dark spot in the left middle is due to a damaged area on the MCP, but it has no significant influence on our results; see text for details.

a $9.5 \mathrm{keV}$ x-ray photon are shown. When the alignment laser was blocked, the image, Figure $3 \mathrm{a}$, shows a practically isotropic velocity distribution for triply charged iodine ions obtained from Coulomb explosion of randomly oriented molecules. As expected for the isotropic sample the measured degree of alignment is $\left\langle\cos ^{2} \theta_{2 \mathrm{D}}\right\rangle=0.50$. Figure $3 \mathrm{~b}-\mathrm{d}$ show the corresponding velocity distributions for triply, singly and doubly charged iodine fragments received from aligned molecules. The majority of detected ions is located at two distinct spots around $\theta_{2 \mathrm{D}}=0$ and $\pi$. The non-isotropic ion fragment distribution is a clear indication that the molecules are aligned along the $X$ axis, the major axis of the polarization ellipse of the alignment laser. The velocity distributions show additional structure due to different fragmentation channels of 2,5-diiodothiophene upon x-ray ionization, but only the areas between the inner and outer white circles were used to determine the degree of alignment. This corresponds to the fastest observed ion fragment for each iodine charge state. These fragments are a good measure of the degree of alignment since they are produced from molecules undergoing the fastest fragmentation, thus exhibiting the most-axial recoil. We point out that non-axial recoil would result in a smaller measured degree of alignment than actually exists and, therefore, that the measurements provide a lower limit for the actual degree of alignment. The resulting degrees of alignment for triply, doubly, and singly charged iodine cations are $\left\langle\cos ^{2} \theta_{2 \mathrm{D}}\right\rangle=0.85$, $\left\langle\cos ^{2} \theta_{2 \mathrm{D}}\right\rangle=0.84$, and $\left\langle\cos ^{2} \theta_{2 \mathrm{D}}\right\rangle=0.82$, respectively. 


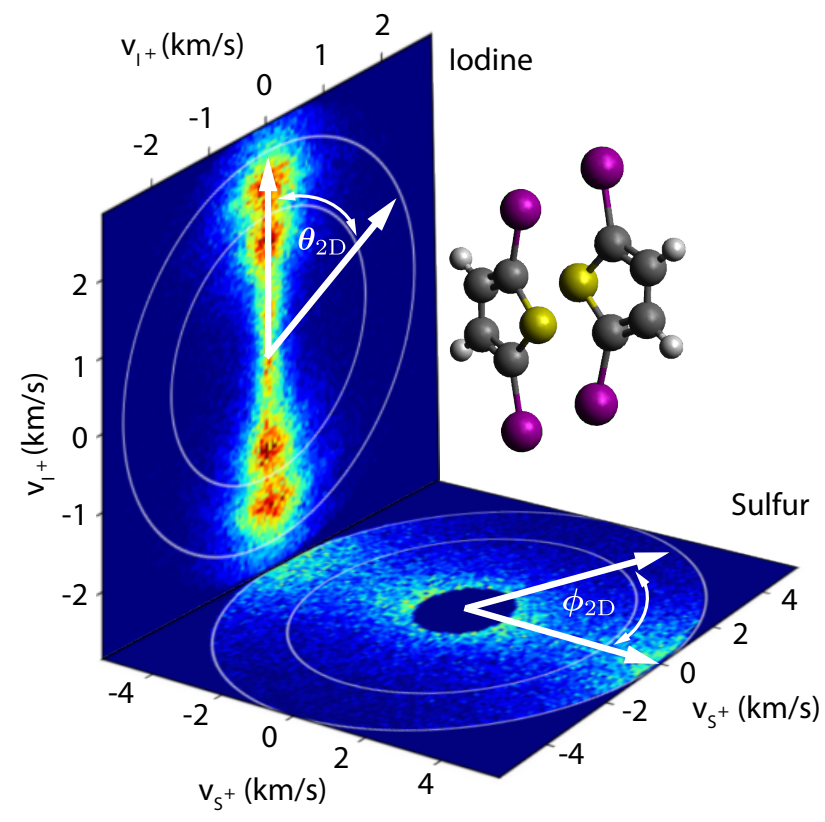

Figure 4. VMI images for singly charged iodine (side view, vertical plane) and sulfur (top view, horizontal plane) measured upon near-infrared strong field ionization. The two molecules sketch the 3D alignment. The area between the inner and outer white circles was used to determine the degree of alignment. Background from low-energy fragmentation channels were removed in the sulfur VMI. The color scale is the same as for Figure 3 The white arrows in the images depict the angles $\theta_{2 \mathrm{D}}$ and $\phi_{2 \mathrm{D}}$.

The dark spot in the left part of the images, best visible in Figure 3 a, is due to a damaged area on the MCP. It has no significant influence on the measured degree of alignment as the velocity distribution of the ions, from aligned molecules, is peaked at $\theta_{2 \mathrm{D}}=0$ and $\pi$. The tilt of the $\mathrm{I}^{+n}$ ion distributions in the VMIs is attributed to imperfect imaging due to a off-center ionization.

\subsection{Results from experiments at CFEL}

Figure 4 shows 2D momentum distributions for singly charged iodine (side view, vertical plane) and sulfur fragments (top view, horizontal plane) upon nearinfrared (NIR) strong-field ionization (SFI) of 3D aligned molecules. The major (minor) axis of the alignment laser polarization ellipse was set along the $X$ axis ( $Y$ axis). The corresponding spatial orientation of $3 \mathrm{D}$ aligned 2,5-diiodothiophene is indicated by the two molecules. For singly charged iodine ions three major Coulomb fragmentation channels are visible by the three maxima in the transverse velocity. The velocity distribution of the fragmentation channel with the highest kinetic energy (indicated by the white circles) was used to determine the degree of alignment as $\left\langle\cos ^{2} \theta_{2 \mathrm{D}}\right\rangle=0.94$, reflecting the very strong alignment of the iodine-iodine axis. For the sulfur ions, two major

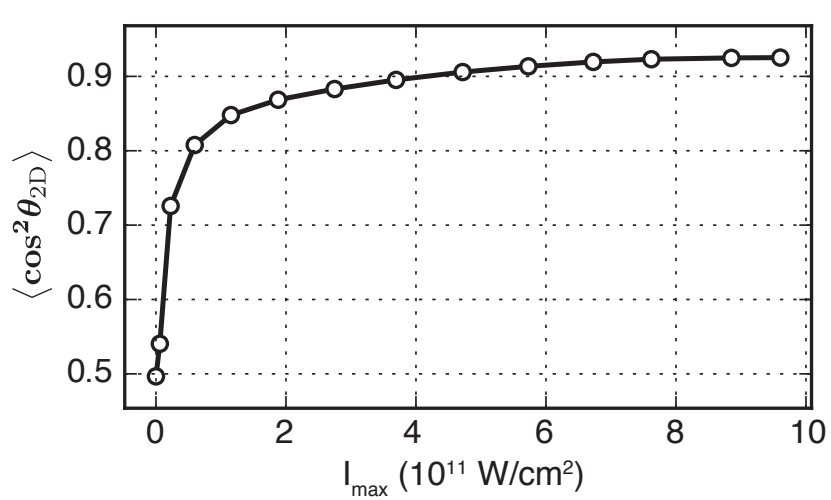

Figure 5. The degree of alignment for singly charged iodine as a function of the peak intensity of the alignment laser using a pulse duration of 485 ps (FWHM). This experiment was conducted at CFEL.

fragmentation channels are observed. The slow channel, located in the center of the image, is significantly stronger in signal and has been removed to increase the contrast for the fast channel. The degree of alignment obtained from the velocity distribution of the fast sulfur ions is $\left\langle\cos ^{2} \phi_{2 \mathrm{D}}\right\rangle=0.79$, reflecting the additional strong alignment of the molecular plane to the plane of polarization of the alignment laser.

In Figure 5 the degree of alignment of the iodineiodine axis is shown as a function of the laser pulse energy, obtained from $\mathrm{I}^{+}$momentum images. The typical power dependence of the degree of alignment for a cold beam is observed. The highest degree of alignment of $\left\langle\cos ^{2} \theta_{2 \mathrm{D}}\right\rangle=0.94$ is obtained at a peak intensity of $9.6 \cdot 10^{11} \mathrm{~W} / \mathrm{cm}^{2}$, but above $7 \cdot 10^{11} \mathrm{~W} / \mathrm{cm}^{2}$ no significant change of the degree of alignment can be seen, indicating the saturation of the degree of alignment.

\section{Discussion}

The principal aim of the experiment performed at LCLS was to record the x-ray diffraction patterns of isolated gas phase molecules, similar to previous experiments [10. 41, which will be reported elsewhere. The experiments at CFEL were performed in preparation of that beamtime. Since in the diffractive imaging experiment the amount of scattered photons scales linearly with the density of the molecules in the molecular beam, the experimental setup was designed such that the pulsed valve was located as close as possible to the interaction region for a collimated, dense, cold molecular beam. A density estimation of the molecular beam can be derived from the mass spectrum, Figure 2. To obtain a lower boundary for the molecular beam density it was assumed that all ionized molecules show two ionic iodine fragments and that the total ion-detection efficiency is $50 \%$. This led to an average of 8.6 ionized molecules 
per x-ray pulse. X-ray photons mainly interact with inner shells electrons and therefore the total molecular photo-absorption cross section is approximated by the sum of the individual atomic photo-absorption cross sections. At a photon energy of $9.5 \mathrm{keV}$ the atomic photo-absorption cross sections are $3.86 \cdot 10^{-20} \mathrm{~cm}^{2}$ for iodine, $3.05 \cdot 10^{-21} \mathrm{~cm}^{2}$ for sulfur, and $4.88 \cdot 10^{-23} \mathrm{~cm}^{2}$ for carbon [48, 49]. The interaction length is given by the FWHM of the molecular beam. This results in a molecular beam density of $1 \cdot 10^{9} \mathrm{~cm}^{-3}$.

To determine the degree of alignment it was assumed that the iodine ions recoil along the most polarizable axis of the molecules, which is parallel to the iodine-iodine axis. This lead to an underestimation of the degree of alignment since positive charges can also be created at different locations in the molecule, for instance at the sulfur atom, leading to a non-axial recoil of the iodine ions. The difference in the degree of alignment between $\mathrm{I}^{+}, \mathrm{I}^{+2}, \mathrm{I}^{+3}$ can be explained by the stronger Coulomb repulsion for higher charge states, resulting in faster fragmentation. This provided a more instantaneous mapping of the molecular orientation. Thus a more accurate, less underestimated, value for the degree of alignment is measured for higher charged states.

For the 3D alignment measurement conducted at CFEL, the difference in alignment of the two different molecular axes $\left(\left\langle\cos ^{2} \theta_{2 \mathrm{D}}\right\rangle\right.$ vs. $\left.\left\langle\cos ^{2} \phi_{2 \mathrm{D}}\right\rangle\right)$ is due to the fact that the most polarizable axis, the iodine-iodine axis, aligns along the long axis of laser polarization, resulting in the strongest interaction. The secondmost polarizable axis of the molecule aligns along the short axis of laser polarization, resulting in a somewhat weaker interaction. For the rigid molecule investigated here, the third axis can safely be assume to be fixed with respect to the two others, and thus shows the same degree of alignment as the secondary axis. We note that all degrees of alignment are specified as projections on the experimental screen, as is typically done in experiments on comparably large and complex molecules [20, 32, 50, 51].

The difference between the measured degree of alignment at LCLS and CFEL can be understood as follows: The major factors that affect the achievable degree of alignment are the peak intensity of the alignment laser pulse, the rise time of the alignment laser pulse, and the rotational temperature of the molecules. Moreover, different probing schemes can influence the observed degree of alignment. Here, the alignment laser pulses, at LCLS and CFEL, are not expected to cause this big discrepancy. Generally, for a given laser peak intensity, the degree of alignment decreases with decreasing adiabaticity. To account for the non-adiabaticity of the molecular alignment, the rise time of the laser pulses and the peak intensities have to be examined. Both laser pulses had a comparable peak intensities in the focus. The rise times at LCLS and CFEL were 70 and 100 ps, respectively. Considering that the largest classical rotation period of the molecule is about $2 \mathrm{~ns}$, it is expected that the alignment dynamics during the rising edges of the laser pulses at both experiments are similar. This is supported by the observed saturation of the degree of alignment for a cold molecular beam, at CFEL, for the investigated peak intensities.

An analysis of the degree of alignment of (complex) molecules in dependence of the pulse duration has not yet been performed, but from other experiments we have learned that the degree of alignment is nearly independent of the pulse duration for a given pulse energy, over a wide range of intermediateregime durations, as long as ionization is avoided 33. Generally, for complex molecules it will be most practical to obtain the optimal pulse duration, for maximum alignment, experimentally and corresponding experiments are forthcoming, especially in preparation of future campaigns at FELs.

The different probing schemes, i. e., single photon x-ray probe at LCLS and IR strong field ionization at CFEL, should both be reliable probing schemes and not cause the discrepancy in the measured degree of alignment. This was confirmed by NIR strong field ionization experiments at LCLS to probe the degree of alignment while no x-rays were available. Here, no significant changes in the degree of alignment compared to the one obtained by the single photon $\mathrm{x}$-ray probe were observed under otherwise identical experimental conditions. Therefore, we conclude that the rotational temperature of the molecular ensemble causes the significant difference in the measured degree of alignment, an effect that was described in detail elsewhere [20, 28]. The temperature of the pulsed valve was approximately $35^{\circ} \mathrm{C}$ higher at LCLS than at CFEL, which leads to a somewhat higher initial rotational temperature in the supersonic expansion. In addition the stagnation pressure of the helium seed gas was lower at LCLS than at CFEL, leading to a less efficient cooling in the expansion. Moreover, at LCLS the pulsed valve was only $18 \mathrm{~cm}$ away from the interaction zone, which is likely too short a distance to get a molecular beam without any collisions between the contained atoms and molecules [52, i. e., the molecular beam could not have reached its terminal temperature.

The achieved degree of alignment is slightly lower than the $\left\langle\cos ^{2} \theta_{2 \mathrm{D}}\right\rangle=0.89$ previously obtained at LCLS for a molecule with a similar polarizability anisotropy [10. This can be attributed to the colder molecular beam achieved in that experiment and the fully adiabatic alignment in the 10-ns-duration pulses provided by a $30 \mathrm{~Hz} \mathrm{Nd}$ :YAG laser system. However, 
while the Nd:YAG laser system needed to be user supplied and required a special setup with significant technical challenges and safety implications, TSLs are generally available at FEL facilities for pumpprobe experiments and typically support the full XFEL repetition rates.

Furthermore, the elliptically polarized laser pulses were expected to $3 \mathrm{D}$ align the molecules. The 3D alignment was not independently measured by ionimaging at LCLS, but demonstrated by the experiments at CFEL using same alignment laser polarization ellipticity. However, due to the warmer molecular beam at LCLS, the molecules are also expected to be less confined in 3D. The final analysis of the x-ray diffraction data will provide more details on this.

The described experiment should be directly extensible to larger (bio)molecules, such as the building blocks of life or small model peptides. These systems often have a polarizability anisotropy comparable to, or even larger than, the current sample and scalar polarizabilities that essentially scale with mass. The main obstacle for the molecular-frame investigation of such systems at FELs is the generation of cold ensembles with sufficient density. Related resolution limits of diffraction experiments had previously been discussed [53].

\section{Summary}

We have shown that it is possible to use the direct output from the regenerative amplifier of the in-house TSL at the CXI beamline at LCLS to strongly align molecules at the full LCLS repetition rate. We have measured a degree of alignment of $\left\langle\cos ^{2} \theta_{2 \mathrm{D}}\right\rangle=0.85$. We reasoned that the degree of alignment was limited by the intrinsic temperature of the molecular beam, which was optimized for increased diffraction signal, rather than by the alignment-laser parameters. We have presented evidence for 3D-alignment of our molecular sample. This allows to directly observe bond length and angles in the molecule in an x-ray diffraction experiment.

The demonstrated technique is advantageous to, for instance, Nd:YAG-laser-based alignment as it allows to utilize the full repetition rate of current FELs. Experiments at those facilities are expensive and by adapting this approach one makes optimal use of the limited beam time. Current FEL facilities already have synchronized TSL systems making the implementation of our setup fast, convenient, and, arguably, easy. Moreover, considering more complex experiments in a fs-laser-pump x-ray-probe scheme, or vice versa, it is possible to use different beams of the same optical laser source as alignment, pump, and probe pulses. This reduces the amount of lasers to be synchronized, the amount of possible sources of errors, and, therefore, makes the experiment overall more stable. Our approach will also allow to utilize a very large fraction, if not all, of the nearly 27000 pulses per second at the upcoming European XFEL or the one million pulses of LCLS-II, solely depending on the availability of a synchronized high-repetition-rate millijoule-level nearinfrared laser system at these facilities. We point out that our method does not rely on the presence of a welldefined molecular recoil-frame and, thus, has advantages over coincidence techniques [54 for molecular-frame investigations of complex molecules, even at high repetition rates. Furthermore, the alignment approach allows for multiple isolated molecules in the interaction volume.

Providing stretched pulses of high-power amplified TSL systems, for instance, with pulse energies on the order of $10 \mathrm{~mJ}$ and durations of $\sim 1 \mathrm{~ns}$, would allow for investigations of strongly-aligned very large molecules, for instance, through coherent diffractive imaging.

\section{Acknowledgments}

Besides DESY, this work has been supported by the Helmholtz Virtual Institute "Dynamic Pathways in Multidimensional Landscapes", the Helmholtz Association "Initiative and Networking Fund", the excellence cluster "The Hamburg Center for Ultrafast Imaging - Structure, Dynamics and Control of Matter at the Atomic Scale" of the Deutsche Forschungsgemeinschaft (CUI, DFG-EXC1074), and the European Research Council through the Consolidator Grant 614507-COMOTION. F. W. acknowledges a Paul Ewald fellowship by the Volkswagen Stiftung. D. R. and A. R. acknowledge support from the Office of Basic Energy Sciences, U. S. Department of Energy. Parts of this research were carried out at the Linac Coherent Light Source (LCLS) at the SLAC National Accelerator Laboratory. LCLS is an Office of Science User Facility operated for the U.S. Department of Energy Office of Science by Stanford University.

\section{References}

[1] Meckel M, Comtois D, Zeidler D, Staudte A, Pavicic D, Bandulet H C, Pepin H, Kieffer J C, Doerner R, Villeneuve D M and Corkum P B 2008 Science 320 1478-1482 URL http://www.sciencemag org/cgi/content/abstract/320/5882/1478

[2] Holmegaard L, Hansen J L, Kalhøj L, Kragh S L, Stapelfeldt H, Filsinger F, Küpper J, Meijer G, Dimitrovski D, Abu-samha M, Martiny C P J and Madsen L B 2010 Nat. Phys. 6428 (Preprint 1003.4634)

[3] Boll R, Anielski D, Bostedt C, Bozek J D, Christensen L, Coffee R, De S, Decleva P, Epp S W, 
Erk B, Foucar L, Krasniqi F, Küpper J, Rouzée A, Rudek B, Rudenko A, Schorb S, Stapelfeldt H, Stener M, Stern S, Techert S, Trippel S, Vrakking M J J, Ullrich J and Rolles D 2013 Phys. Rev. A $88061402(\mathrm{R})$

[4] Itatani J, Levesque J, Zeidler D, Niikura H, Pépin H, Kieffer J C, Corkum P B and Villeneuve D M 2004 Nature 432 867-871

[5] Wörner H J, Bertrand J B, Kartashov D V, Corkum P B and Villeneuve D M 2010 Nature 466 604-607

[6] Zuo T, Bandrauk A D and Corkum P B 1996 Chem. Phys. Lett. 259 313-320

[7] Blaga C I, Xu J, DiChiara A D, Sistrunk E, Zhang K, Agostini P, Miller T A, DiMauro L F and Lin C D 2012 Nature 483 194-197

[8] Hensley C J, Yang J and Centurion M 2012 Phys. Rev. Lett. 109133202

[9] Sciaini G and Miller R J D 2011 Rep. Prog. Phys. 74096101 URL http://iopscience.iop.org/ 0034-4885/74/9/096101/

[10] Küpper J, Stern S, Holmegaard L, Filsinger F, Rouzée A, Rudenko A, Johnsson P, Martin A V, Adolph M, Aquila A, Bajt S, Barty A, Bostedt C, Bozek J, Caleman C, Coffee R, Coppola N, Delmas T, Epp S, Erk B, Foucar L, Gorkhover T, Gumprecht L, Hartmann A, Hartmann R, Hauser G, Holl P, Hömke A, Kimmel N, Krasniqi F, Kühnel K U, Maurer J, Messerschmidt M, Moshammer R, Reich C, Rudek B, Santra R, Schlichting I, Schmidt C, Schorb S, Schulz J, Soltau H, Spence J C H, Starodub D, Strüder L, Thøgersen J, Vrakking M J J, Weidenspointner G, White T A, Wunderer C, Meijer G, Ullrich J, Stapelfeldt H, Rolles D and Chapman H N 2014 Phys. Rev. Lett. 112083002 (Preprint 1307.4577)

[11] Barty A, Küpper J and Chapman H N 2013 Annu. Rev. Phys. Chem. 64 415435 URL http://dx.doi.org/10.1146/ annurev-physchem-032511-143708

[12] Stern S, Chapman H and Küpper J 2015 J. Phys. $B$ In preparation for special issue on "science at free-electron lasers"

[13] Stapelfeldt H and Seideman T 2003 Rev. Mod. Phys. 75 543-557 URL http://link.aps.org/ abstract/RMP/v75/p543

[14] Filsinger F, Meijer G, Stapelfeldt H, Chapman H and Küpper J 2011 Phys. Chem. Chem. Phys. 13 2076-2087

[15] Reuss J 1988 State Selection by Nonoptical Methods Atomic and molecular beam methods vol 1 ed Scoles G (New York, NY, USA: Oxford
University Press) chap 11, pp 276-292 ISBN 0195042808

[16] Block P A, Bohac E J and Miller R E 1992 Phys. Rev. Lett. 68 1303-1306

[17] Normand D, Lompre L and Cornaggia C $1992 \mathrm{~J}$. Phys. B 25 497-503

[18] Rosca-Pruna F and Vrakking M J J 2001 Phys. Rev. Lett. 87153902 URL http://prola.aps org/abstract/PRL/v87/i15/e153902

[19] Sakai H, Safvan C P, Larsen J J, Hilligsøe K M, Hald K and Stapelfeldt H 1999 J. Chem. Phys. 110 10235-10238 URL http://link.aip.org/link/ ? JCP/110/10235/1

[20] Holmegaard L, Nielsen J H, Nevo I, Stapelfeldt H, Filsinger F, Küpper $\mathrm{J}$ and Meijer G 2009 Phys. Rev. Lett. 102(2) 023001 (Preprint 0810.2307) URL http://dx.doi.org/10.1103/ PhysRevLett.102.023001

[21] Ghafur O, Rouzee A, Gijsbertsen A, Siu W K, Stolte S and Vrakking M J J 2009 Nat. Phys. 5 289-293

[22] Filsinger F, Küpper J, Meijer G, Holmegaard L, Nielsen J H, Nevo I, Hansen J L and Stapelfeldt H 2009 J. Chem. Phys. 131064309 (Preprint 0903.5413) URL http://scitation.aip.org/content/aip/ journal/jcp/131/6/10.1063/1.3194287

[23] Larsen J J, Hald K, Bjerre N, Stapelfeldt H and Seideman T 2000 Phys. Rev. Lett. 85 2470-2473

[24] Lee K F, Villeneuve D M, Corkum P B, Stolow A and Underwood J G 2006 Phys. Rev. Lett. 97 173001 URL http://link.aps.org/abstract/ PRL/v97/e173001

[25] Ren X, Makhija V and Kumarappan V 2014 Phys. Rev. Lett. 112173602

[26] Tanji H, Minemoto S and Sakai H 2005 Phys. Rev. A 72063401

[27] Pabst S, Ho P J and Santra R 2010 Phys. Rev. A 81 043425 URL http://pra.aps.org/abstract/ PRA/v81/i4/e043425

[28] Kumarappan V, Bisgaard C Z, Viftrup S S, Holmegaard L and Stapelfeldt H 2006 J. Chem. Phys. 125194309

[29] Ortigoso J, Rodriguez M, Gupta M and Friedrich B 1999 J. Chem. Phys. 110 3870-3875 URL http://link.aip.org/link/?JCP/110/3870/1

[30] Torres R, de Nalda R and Marangos J P 2005 Phys. Rev. A 72023420

[31] Owschimikow N, Schmidt B and Schwentner N 2011 Phys. Chem. Chem. Phys. 13(19) 8671-8680 
[32] Trippel S, Mullins T, Müller N L M, Kienitz J S, Długołęcki K and Küpper J 2013 Mol. Phys. 111 1738 (Preprint 1301.1826)

[33] Trippel S, Mullins T, Müller N L M, Kienitz J S, Omiste J J, Stapelfeldt H, González-Férez $\mathrm{R}$ and Küpper J 2014 Phys. Rev. A 89 051401(R) (Preprint 1401.6897)

[34] Boutet S and Williams G 2010 New J. Phys. 12 035024

[35] Liang M, Williams G J, Messerschmidt M, Seibert M M, Montanez P A, Hayes M, Milathianaki D, Aquila A, Hunter M S, Koglin J E, Schafer D W, Guillet S, Busse A, Bergan R, Olson W, Fox K, Stewart N, Curtis R, Miahnahri A A and Boutet S 2015 J. Synchrotron Rad. 22 514-519

[36] Johnsson P, Rouzée A, Siu W, Huismans Y, Lepine F, Marchenko T, Dusterer S, Tavella F, Stojanovic N, Azima A, Treusch R, Kling M F and Vrakking M J J 2009 J. Phys. B 42 134017 URL http: //www . iop.org/EJ/abstract/ 0953-4075/42/13/134017/

[37] Glownia J M, Cryan J, Andreasson J, Belkacem A, Berrah N, Blaga C I, Bostedt C, Bozek J, DiMauro L F, Fang L, Frisch J, Gessner O, Guehr M, Hajdu J, Hertlein M P, Hoener M, Huang G, Kornilov O, Marangos J P, March A M, McFarland B K, Merdji H, Petrovic V S, Raman C, Ray D, Reis D A, Trigo M, White J L, White W, Wilcox R, Young L, Coffee R N and Bucksbaum P H 2010 Opt. Exp. 18 17620-17630

[38] Cryan J P, Glownia J M, Andreasson J, Belkacem A, Berrah N, Blaga C I, Bostedt C, Bozek J, Buth C, DiMauro L F, Fang L, Gessner O, Guehr M, Hajdu J, Hertlein M P, Hoener M, Kornilov O, Marangos J P, March A M, McFarland B K, Merdji H, Petrovic V S, Raman C, Ray D, Reis D, Tarantelli F, Trigo M, White J L, White W, Young L, Bucksbaum P H and Coffee R N 2010 Phys. Rev. Lett. 105083004

[39] Rolles D, Boll R, Adolph M, Aquila A, Bostedt C, Bozek J, Chapman H, Coffee R, Coppola N, Decleva P, Delmas T, Epp S, Erk B, Filsinger F, Foucar L, Gumprecht L, Hömke A, Gorkhover T, Holmegaard L, Johnsson P, Kaiser C, Krasniqi F, Kühnel K U, Maurer J, Messerschmidt M, Moshammer R, Quevedo W, Rajkovic I, Rouzée A, Rudek B, Schlichting I, Schmidt C, Schorb S, Schröter C D, Schulz J, Stapelfeldt H, Stener M, Stern S, Techert S, Thøgersen J, Vrakking M J J, Rudenko A, Küpper J and Ullrich J 2014 J. Phys. B 47124035

[40] Boll R, Rouzée A, Adolph M, Anielski D, Aquila A, Bari S, Bomme C, Bostedt C, Bozek J D, Chapman H N, Christensen L, Coffee R, Coppola N, De S,
Decleva P, Epp S W, Erk B, Filsinger F, Foucar L, Gorkhover T, Gumprecht L, Hömke A, Holmegaard L, Johnsson P, Kienitz J S, Kierspel T, Krasniqi F, Kühnel K U, Maurer J, Messerschmidt M, Moshammer R, Müller N L M, Rudek B, Savelyev E, Schlichting I, Schmidt C, Scholz F, Schorb S, Schulz J, Seltmann J, Stener M, Stern S, Techert S, Thøgersen J, Trippel S, Viefhaus J, Vrakking M, Stapelfeldt H, Küpper J, Ullrich J, Rudenko A and Rolles D 2014 Faraday Disc. 171 57-80 (Preprint 1407.7782)

[41] Stern S, Holmegaard L, Filsinger F, Rouzee A, Rudenko A, Johnsson P, Martin A V, Barty A, Bostedt C, Bozek J, Coffee R, Epp S, Erk B, Foucar L, Hartmann R, Kimmel N, Kühnel K U, Maurer J, Messerschmidt M, Rudek B, Starodub D, Thøgersen J, Weidenspointner G, White T A, Stapelfeldt H, Rolles D, Chapman H N and Küpper J 2014 Faraday Disc. 171393 (Preprint 1403.2553)

[42] Even U, Jortner J, Noy D, Lavie N and CossartMagos N 2000 J. Chem. Phys. 112 8068-8071

[43] Barty A, Kirian R A, Maia F R N C, Hantke M, Yoon C H, White T A and Chapman H $2014 \mathrm{~J}$. Appl. Crystallogr. $471118-1131$

[44] Kella D, Algranati M, Feldman H, Heber O, Kovner H, Malkin E, Miklazky E, Naaman R, Zajfman D, Zajfman J and Vager Z 1993 Nucl. Instrum. Meth. A 329 440-452 ISSN 0168-9002

[45] Chang B Y, Hoetzlein R C, Mueller J A, D G J and Houston P L 1998 Rev. Sci. Instrum. 69(4) 1665-1670

[46] Wiley W and Mclaren I 1955 Rev. Sci. Instrum. 26 1150-1157

[47] Eppink A T J B and Parker D H 1997 Rev. Sci. Instrum. 68 3477-3484 URL http://link.aip org/link/?RSI/68/3477/1

[48] Stern S 2013 Controlled Molecules for X-ray Diffraction Experiments at FreeElectron Lasers Dissertation Universität Hamburg Hamburg, Germany URL http: //www.desy.de/ stern/PhD_StephanStern_ SubmittedVersion_2013-10-18.pdf

[49] Berger M, Hubbell J, Seltzer S, Chang J, Coursey J, Sukumar R, Zucker D and Olsen K 2010 XCOM: Photon Cross Section Database (version 1.5) URL http://physics.nist.gov/xcom

[50] Larsen J J, Sakai H, Safvan C P, Wendt-Larsen I and Stapelfeldt H 1999 J. Chem. Phys. 1117774

[51] Hansen J L, Omiste Romero J J, Nielsen J H, Pentlehner D, Küpper J, González-Férez R and Stapelfeldt H 2013 J. Chem. Phys. 139234313 
(Preprint 1308.1216) URL http://arxiv.org/ abs/1308.1216

[52] Luria K, Christen W and Even U 2011 J. Phys. Chem. A 115 7362-7367

[53] Spence J C H, Schmidt K, Wu J S, Hembree G, Weierstall U, Doak R B and Fromme P 2005 Acta Cryst. A 61 237-245 URL http://dx.doi.org/ 10.1107/S0108767305002710

[54] Ullrich J, Moshammer R, Dorn A, Dörner R, Schmidt L P H and Schmidt-Böcking H 2003 Rep. Prog. Phys. 66 1463-1545 URL http://stacks iop.org/0034-4885/66/1463 